\title{
Influence of Frequency Ratio on the Hydroelastic Response of a Cylinder with Degrees of Freedom under Vortex Induced Vibration
}

\author{
Vidya Chandran, M Sekar, Sheeja Janardhanan
}

\begin{abstract}
Vortex induced vibration of cylindrical structures is an extensively researched topic. Most of the studies have concentrated on the response of the cylinder in the cross flow $(C F)$ direction. In a realistic ocean environment, structures such as drilling and marine risers are more or less free to vibrate both in CF and in line (IL) directions. It has also been observed that the $I L$ vibrations have significant influence on the $C F$ response. Interaction between the responses in inline and cross flow directions has still been not fully understood. This paper addresses the same through a simplified numerical method for understanding the interaction between these two responses using two dimensional computational fluid dynamics (CFD) simulations. Here analyzes two cases have been considered; where in the cylinder is modeled with two different values of ratio of natural frequency of the cylinder in the IL direction to that in the $C F$ direction. The trends of variation of hydrodynamic and structural parameters have been analyzed to comprehend the effect of directional natural frequency ratio on the cylinder response and hydrodynamic force coefficients. The shedding pattern has also been studied in this paper. An increase by $18 \%$ in the value of the lift coefficient and $38 \%$ of that in the drag coefficient has been observed when the frequency ratio is increased from 1 to 2. The results show that the cylinder with frequency ratio 2 is more prone to lock in vibration. This phenomenon may be related to the shifting of shedding pattern from $2 S$ to $P+S$ mode when the frequency ratio is 2 .
\end{abstract}

Index Terms: CFD, cross flow vibration, hydroelastic response, inline vibration, vortex induced vibration.

\section{INTRODUCTION}

Vortex induced vibration (VIV) of marine risers has ever been quite an enticing topic for researchers. However most of the studies have concentrated on understanding the wake characteristics and estimating the hydrodynamic loading and the subsequent response of either stationary cylinder or cylinder with single degree of freedom (SDOF) [1]. The results on the study of hydrodynamic response of cylinder with TDOF in both in-line (IL) and cross-flow (CF) directions are scanty. IL vibration has significant impact on the shedding pattern and also on the amplitude of $\mathrm{CF}$ vibrations [2]. The first of its kind discussions were reported in the case of flow around cylinder with TDOF [3], Establishing the effect of reduced velocity $\left(U_{\mathrm{r}}\right)$ on forced and

Revised Manuscript Received on July 22, 2019.

Vidya Chandran, Department of Mechanical Engineering, Karunya Institute of Technology and sciences, Coimbatore, India

M Sekar, Department of Mechanical Engineering, AAA College of Engineering and Technology, Sivakasi, India.

Sheeja Janardhanan, Department of Mechanical Engineering, SCMS School of Engineering and Technology, Ernakulum, India. free TDOF cylinder response [3]. The effect of IL response on $\mathrm{CF}$ response depends on the ratio of natural frequencies in both the directions $\left(\eta_{\mathrm{b}}=\frac{f_{\mathrm{nIL}}}{f_{\mathrm{nCF}}}\right)$, where $\mathrm{f}_{\mathrm{nCF}}$ is the natural frequency of the cylinder in the CF direction and $\mathrm{f}_{\mathrm{nIL}}$ is the same in the IL direction. During lock in, if the natural frequency in the IL direction is twice that in the CF direction, resonance occurs in both directions leading to premature failure of the riser [4]. This aspect of VIV needs a detailed study to understand the influence of natural frequency ratio on the shedding pattern and hydrodynamic force coefficients. Also it has been observed that IL response amplitude is a function of the reduced velocity, $\mathrm{U}_{\mathrm{r}}$ and stability parameters, whereas CF response amplitude is a function of $U_{\mathrm{r}}$ and flow velocity [5]. Wake characteristics, hydrodynamic force coefficients as well as the structural response vary significantly with frequency ratio. Hence there is a need to develop a simplified method for the prediction of response that holds good for different values of $\eta_{\mathrm{b}}$.

\section{PROBLEM DESCRIPTION}

In the present paper a riser model with outer diameter $0.076 \mathrm{~m}$ has been numerically analyzed using two dimensional (2D) Reynolds average Navier-Strokes (RANS) based computational fluid dynamics (CFD) approach. Specifications of the riser and the flow conditions are listed in Table 1. Table 1 Riser model specifications and flow characteristics

\begin{tabular}{|c|c|c|}
\hline Properties & Values & Units \\
\hline Diameter of the Cylinder $(D)$ & 0.076 & $\mathrm{~m}$ \\
\hline $\begin{array}{c}\text { Aspect ratio of Cylinder }( \\
L / D)\end{array}$ & 13.12 & - \\
\hline Inlet Velocity $(V)$ & 0.5 & $\mathrm{~m} / \mathrm{s}$ \\
\hline Flow Reynolds Number $(\mathrm{Re})$ & $3.8 \times 10^{4}$ & - \\
\hline $\begin{array}{c}\text { Mass ratio of the Cylinder } \\
\left(m^{*}\right)\end{array}$ & 0.66 & - \\
\hline
\end{tabular}

The incoming flow velocity is taken as $0.5 \mathrm{~m} / \mathrm{s}$ to maintain the flow regime uniform at $\mathrm{Re}=3.8 \times 10^{4}$ which corresponds to the average current velocity in the ocean flow condition frequently encountered by a real marine riser used for petroleum extraction in offshore industries [6]. In this paper an effort has been made to study the influence of ratio of natural frequencies in the $\mathrm{CF}$ and IL directions on the wake dynamics. 


\section{MATHEMATICAL MODEL}

The riser has been modeled as a 2D cylinder with TDOF in the CF and IL directions. The equations of motion for the riser can be represented as in Equations (1) and (2)

$$
\begin{aligned}
& \mathrm{m} \ddot{x}+c \dot{x}+k x=F_{D}(t) \\
& m \ddot{y}+c \dot{y}+k y=F_{L}(t)
\end{aligned}
$$

where $\mathrm{x}$ is the displacement in IL direction and $y$ is the displacement in the $\mathrm{CF}$ direction. The excitation forces are drag force, $\mathrm{F}_{\mathrm{D}}(t)$ and lift force $\mathrm{F}_{\mathrm{L}}(t)$ represented as in Equations (3) and (4)

$$
\begin{aligned}
& F_{D}=C_{D} \frac{1}{2} \rho A V^{2} \\
& F_{L}=C_{L} \frac{1}{2} \rho A V^{2}
\end{aligned}
$$

$C_{\mathrm{D}}$ is the coefficient of drag and $C_{\mathrm{L}}$ is the coefficient of lift.

The periodic excitation forces caused by the alternate shedding of vortices, make the riser oscillate in CF as well as IL directions. The riser is observed to oscillate with frequency equal to frequency of vortex shedding $\left(f_{\mathrm{v}}\right)$ in the $\mathrm{CF}$ direction and at double the frequency in the IL direction during lock In [7]. Lock in can be defined as the resonance condition during which the vortex shedding frequency locks on to the natural frequency of the riser in the cross flow direction [8]. A simple representation of the mathematical model of riser with TDOF is represented in Figure.1 [9], [10].

The structural damping component is neglected in the model in both CF and IL directions, considering its trivial nature in the present model. $k_{\mathrm{x}}$ and $k_{\mathrm{y}}$ are stiffness coefficients in the IL and CF directions respectively. In the present study $k_{\mathrm{x}}$ and $k_{\mathrm{y}}$ are varied in the user defined function (UDF) hooked to the dynamic boundary of the cylinder to achieve various cases of frequency ratios.

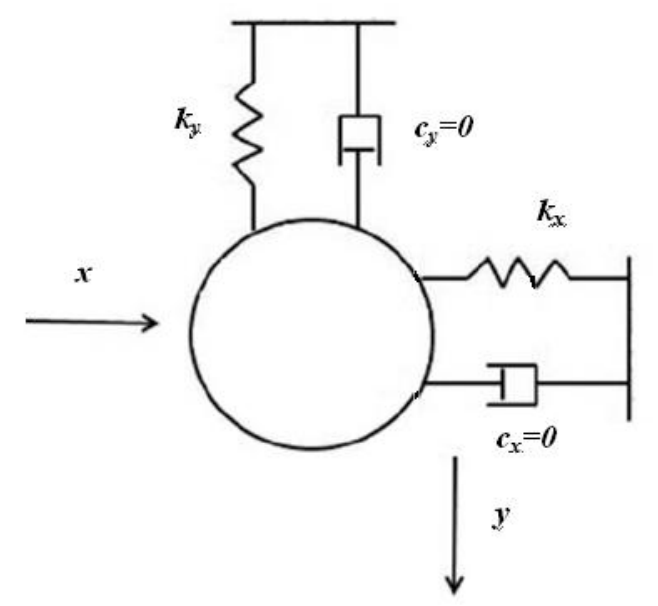

Figure 1: Representation of mathematical model of riser with TDOF.

\section{FLOW MODEL}

Figure 2 (a) shows the computational domain for the present analysis. Total length of the fluid domain for analysis is $40 \mathrm{D}$. $\mathrm{D}$ is the diameter of the cylinder. The cylinder located not at the centre of the domain but $10 \mathrm{D}$ away from the inlet boundary. Width of the symmetric fluid domain is $10 \mathrm{D}$ on both sides of the central flow line with the cylinder located at the middle. A representation of the computational domain and the mesh around the oscillating cylinder has been shown in Figure 2 (c) and (b) respectively. From boundary layer theory, thickness of boundary layer on the cylindrical surface is obtained [11]. To ensure that vortex shedding, which is a boundary layer phenomenon, is captured accurately, near wall element size has been calculated considering the boundary layer thickness. With 307 nodes on the cylinder circumference, minimum element size near the cylinder, which is modeled as a rigid body is obtained to be $0.0001 D$. Value of $y+$ otherwise known as non-dimensional element size near the rigid cylinder boundary is found to be within the permissible range $(<1)$. The oscillating cylinder is assumed to have smooth surface. On the cylinder wall no slip boundary condition has been applied and on the inlet boundary, velocity-inlet boundary condition with inflow at velocity $=0.5 \mathrm{~m} / \mathrm{s}$. Pressure outlet boundary condition has been applied to the outlet boundary of the domain. Fluid velocity gradient at the outlet is set to be zero and the pressure as zero reference pressure. The two transverse boundaries are treated as symmetry boundary conditions. Grid independency study has already been carried out for the present grid and has been reported in the previous work done by the authors [12].

The numerical analysis of the cylinder oscillating under VIV has been performed by treating the problem as a case of two way fluid structure interactions (2way FSI). ANSYS ICEM CFD has been used for Modeling and meshing of the cylinder and domain. For flow analysis ANSYS FLUENT flow solver has been used. Flow past the dynamic rigid cylinder is solved using the transient, incompressible Navier-Stokes equation based RANS solver. $\mathrm{k}-\omega$ SST has been chosen as the turbulence model, since this model was proved to be the best turbulence model for prediction of VIV [13]. RANS solver for transient analysis can be explained as follows using Equation (5) and (6).

$$
\begin{gathered}
\frac{\partial \rho}{\partial \mathrm{t}}+\frac{\partial}{\partial \mathrm{x}_{\mathrm{i}}}\left(\rho \mathrm{u}_{\mathrm{i}}\right)=0 \\
\frac{\partial}{\partial \mathrm{t}}\left(\rho \mathrm{u}_{\mathrm{i}}\right)+\frac{\partial}{\partial \mathrm{x}_{\mathrm{i}}}\left(\rho \mathrm{u}_{\mathrm{i}} \mathrm{u}_{\mathrm{j}}\right) \\
=\frac{\partial \mathrm{p}}{\partial \mathrm{x}_{\mathrm{i}}} \\
+\frac{\partial}{\partial \mathrm{x}_{\mathrm{j}}}\left[\mu \left(\frac{\partial \mathrm{u}_{\mathrm{i}}}{\partial \mathrm{x}_{\mathrm{j}}}+\frac{\partial \mathrm{u}_{\mathrm{j}}}{\partial \mathrm{x}_{\mathrm{i}}}\right.\right. \\
\left.\left.-\frac{2}{3} \delta_{\mathrm{ij}} \frac{\partial \mathrm{u}_{\mathrm{l}}}{\partial \mathrm{x}_{\mathrm{l}}}\right)\right]+\frac{\partial}{\partial \mathrm{x}_{\mathrm{j}}}\left(-\rho \overline{\mathrm{u}_{\mathrm{i}}^{\prime} \mathrm{u}_{\mathrm{j}}^{\prime}}\right)
\end{gathered}
$$

Finite difference method discretization of these governing equations is done by the flow solver. A novel approach for optimization of computing time for dynamic mesh simulation is adopted by using non-iterative time advancement (NITA) scheme. Fractional time stepping method (FSM) for pressure velocity coupling is used in order to avoid extreme mesh fineness. This method enables the solver to considerably reduce computational effort and time. 


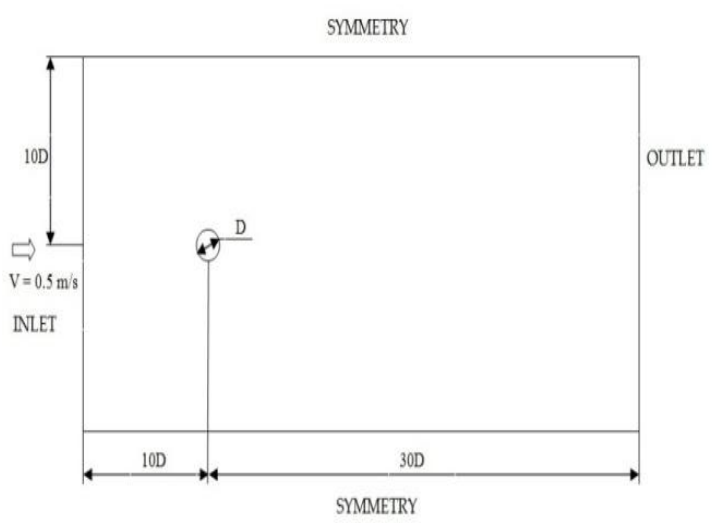

Figure 2 (a): Computational domain

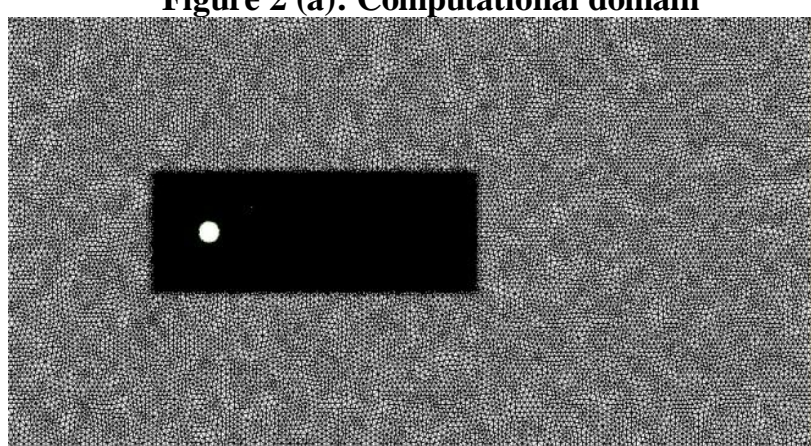

Figure 2 (b): computational mesh

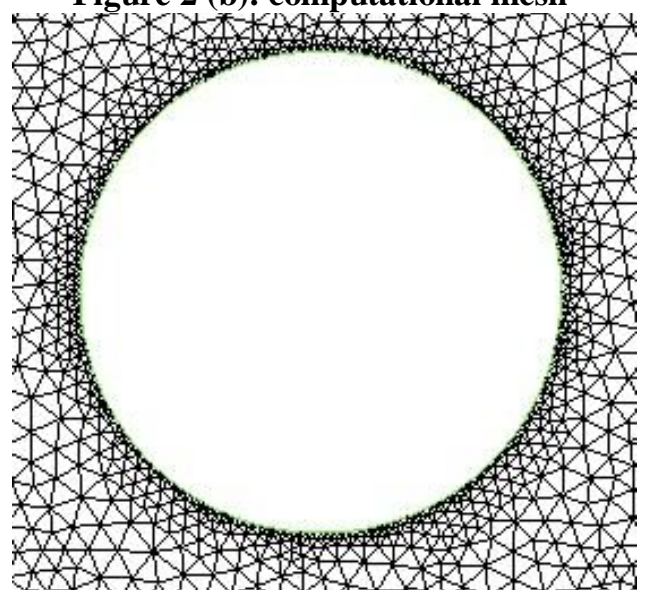

Figure 2 (c): mesh around the cylinder

\section{STRUCTURAL MODEL}

A six degrees of freedom solver (6DOF) is employed to solve the equations of motion both in CF and IL directions. The $6 \mathrm{DOF}$ solver is an integral part of the main flow solver. The $6 \mathrm{DOF}$ solver defines the cylinder as a rigid body capable to move in the $\mathrm{CF}$ and IL directions. The cylinder rigid boundary is made dynamic by hooking on to it a user defined function (UDF) compiled in $\mathrm{C}$ programming language. In the first case where $\eta_{\mathrm{b}}=1$, stiffness coefficient in both CF and IL directions are taken to be equal to $338 \mathrm{~N} / \mathrm{m}$ which corresponds to reduced velocity $\mathrm{U}_{r}=5$ and natural frequency of the cylinder in $\mathrm{CF}$ and IL direction $f_{\mathrm{nCF}}=$ $f_{\mathrm{nIL}}=1.3$. While in the second case, $\eta_{\mathrm{b}}=2$ is obtained by giving values $k_{\mathrm{x}}=1355 \mathrm{~N} / \mathrm{m}$ and $k_{\mathrm{y}}=338 \mathrm{~N} / \mathrm{m}$ in the UDF. Reduced velocity based on natural frequency in the $\mathrm{CF}$ direction and the natural frequency of the cylinder in the $\mathrm{CF}$ direction remains same as the previous case with $\mathrm{U}_{r}=5$ and $f_{\mathrm{nCF}}=1.3$, but the natural frequency in the IL direction doubles showing $f_{\mathrm{nIL}}=2.6$. From previous analysis it has been observed at $\mathrm{U}_{r}=5, \eta=1$ [12]. This corresponds to matching of vortex shedding frequency and natural frequency of cylinder otherwise called lock in phenomenon. The governing equations for the motion of the center of gravity of the cylinder in the CF and IL directions are solved in the inertial coordinate system. Velocity in the CF and IL directions are obtained by performing integration on Eq. (7) and (8)

$$
\begin{aligned}
& \ddot{x}=\frac{1}{m} \sum F_{\mathrm{D}} \\
& \ddot{y}=\frac{1}{m} \sum F_{\mathrm{L}}
\end{aligned}
$$

where $\ddot{x}$ and $\ddot{y}$, are accelerations in the CF and IL direction respectively, $m$ is the sum of the mass of the cylinder and its added mass, $F$, resultant fluid force acting on the cylinder in the respective direction. Equations of motion of the spring mass system given by Eq. (1) and (2) are solve to update the center of gravity of the cylinder.

\section{RESULTS \& DISCUSSION}

From the TDOF analysis it is observed that the characteristics of hydrodynamic force vary significantly with natural frequency ratio. With $\eta_{b}=1, C_{L}$ oscillates uniformly about zero value and the maximum is observed as 0.69 . But with $\eta_{b}=2, C_{L}$ shows non-uniform and irregular oscillation. Maximum amplitude of $\mathrm{C}_{\mathrm{L}}$ varies in the positive and negative direction with an average of 0.85 . A beating pattern is observed in the time history of $C_{L}$ for the latter case. $C_{D}$ shows a commendable increase when $\eta_{b}=2 . C_{D}$ rms value increases from 1.43 to 2.19 when frequency ratio is varied from 1 to 2 . Magnitudes of hydrodynamic parameters are significantly influenced by the natural frequency of the structure. In both cases the stiffness coefficient and hence the natural frequency remains constant in the $\mathrm{CF}$ direction. It is observed that $C_{L}$ value for both the cases are in comparable range. But the natural frequency of oscillation in the IL direction has been doubled in the second case than in the first case. The value of drag coefficient has also significantly increased which may be considered as the flow response to the increase in natural frequency. Important hydrodynamic and structural parameters are given in Table 2. Results of oscillation amplitude obtained with frequency ratio 1 are verified using results from previous numerical studies [14].

Oscillation frequency of $\mathrm{C}_{\mathrm{L}}$ is found to deviate from theoretical value of vortex shedding frequency $f_{\mathrm{v}}=1.3$, estimated from St $=0.2$ when $\eta_{\mathrm{b}}=1$. With $\eta_{\mathrm{b}}=2, f_{\text {osc }}$, has almost tripled to 3.56. Frequency of oscillation of drag coefficient slightly decreases to 2.11 from 2.32 with $\eta_{\mathrm{b}}=2$. Frequency of $\mathrm{CF}$ oscillation remains same for both cases even if the force variation shows different pattern. With $\eta_{\mathrm{b}}=1$, IL oscillation frequency of cylinder is thrice that of CF oscillation frequency. With $\eta_{\mathrm{b}}=2$, IL oscillation frequency of cylinder is twice that of $\mathrm{CF}$ oscillation frequency which is more realistic with the condition $\eta_{\mathrm{b}}=2$. The oscillation frequency in both CF and IL direction in this case is closer to the natural frequency in the respective directions compared to $\eta_{\mathrm{b}}=1$ case. 
Influence of Frequency Ratio on the Hydroelastic Response of a Cylinder with Degrees of Freedom under Vortex Induced Vibration

Table 2 Hydrodynamic and structural parameter of cylinder

\begin{tabular}{|l|c|c|}
\hline Parameters & $\eta_{\mathrm{b}}=1$ & $\eta_{\mathrm{b}}=2$ \\
\hline$C_{\mathrm{L}}$ & 0.69 & 0.85 \\
\hline$C_{\mathrm{D}}$ & 1.43 & 2.19 \\
\hline$f_{\text {osc }} \mathrm{C}_{\mathrm{L}}$ & 1.14 & 3.56 \\
\hline$f_{\text {osc }} \mathrm{D}$ & 2.32 & 2.11 \\
\hline$f_{\mathrm{CF}}$ & 1.15 & 1.13 \\
\hline$f_{\mathrm{IL}}$ & 3.28 & 2.24 \\
\hline$S t$ & 0.17 & 0.17 \\
\hline$Y / D$ & 1.2 & 0.45 \\
\hline$X / D$ & 0.17 & 0.47 \\
\hline
\end{tabular}

When $\eta_{\mathrm{b}}=2$, the non dimensional amplitude Y/D is 1.2 and $\mathrm{X} / \mathrm{D}$ is 0.17 . With $\eta_{\mathrm{b}}=2$, the oscillation in CF direction is observed to be non uniform and irregular. IL oscillations show a more uniform pattern. The most significant observation is that the cylinder oscillates both in IL and CF direction with almost same amplitude of oscillation unlike the oscillations during $\eta_{\mathrm{b}}=1$. Time histories of force coefficients and non dimensional oscillation amplitudes are represented in Figure 3.

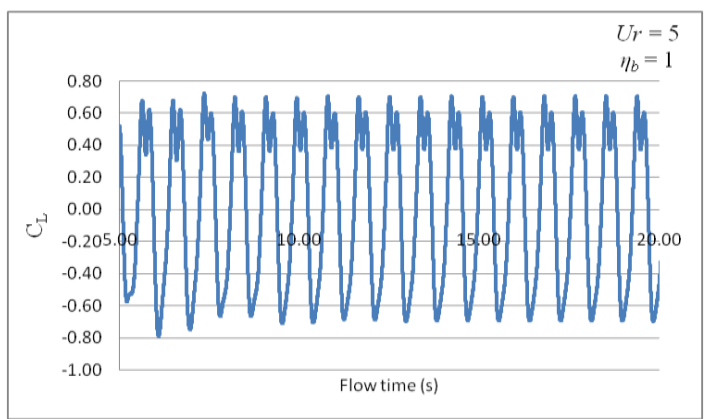

(a)

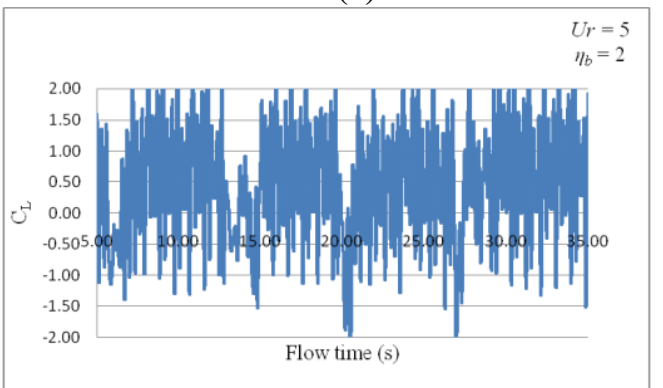

(b)

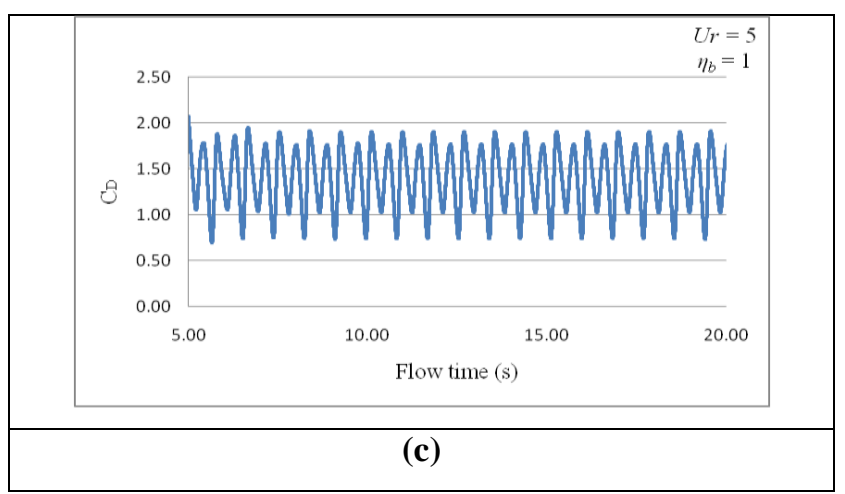

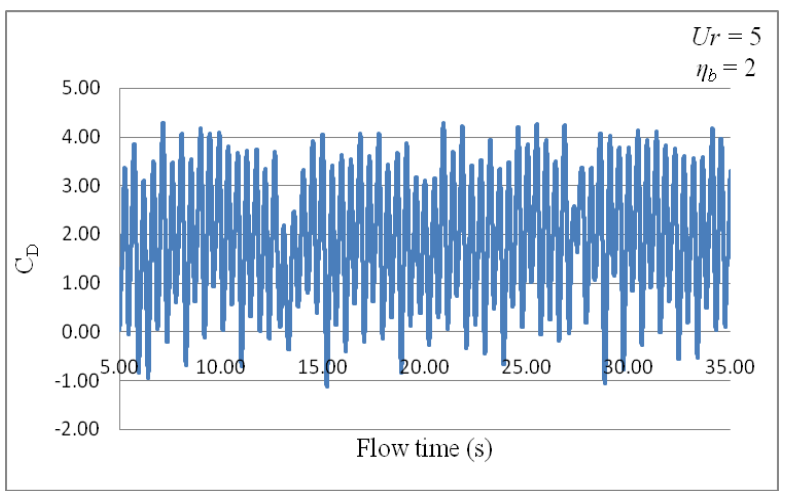

(d)
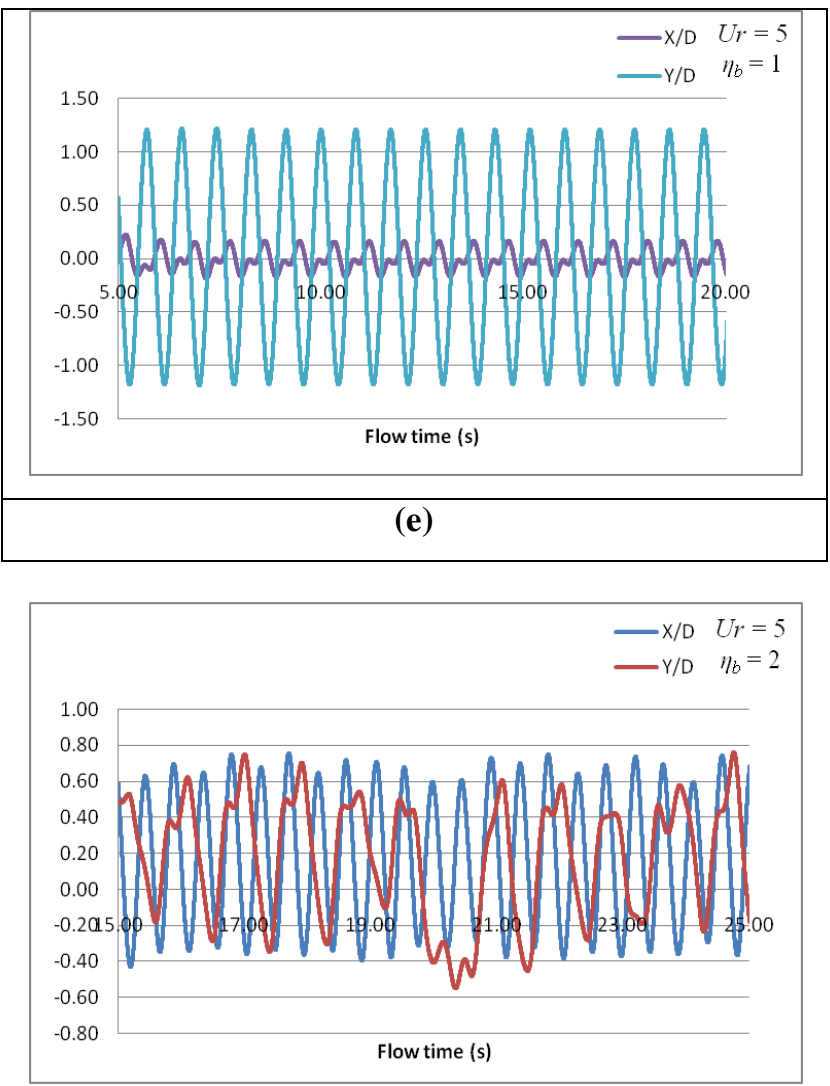

(f)

Figure 3: Pressure contours and Time histories of various hydrodynamic and structural parameters (a)

$C_{L}$ of cylinder with TDOF at $\eta_{b}=1$ (b) $C_{L}$ of cylinder with TDOF at $\eta_{b}=2$ (c) $C_{D}$ of cylinder with TDOF at $\eta_{b}=1$ (d) $C_{D}$ of cylinder with TDOF at $\eta_{b}=2$ (e)motion historyof cylinder with TDOF at $\eta_{b}=1$ (f) motion history of cylinder with TDOF at $\eta_{\mathrm{b}}=2$

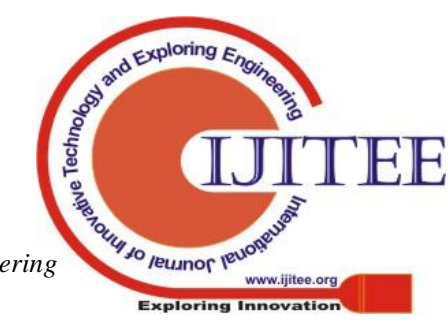




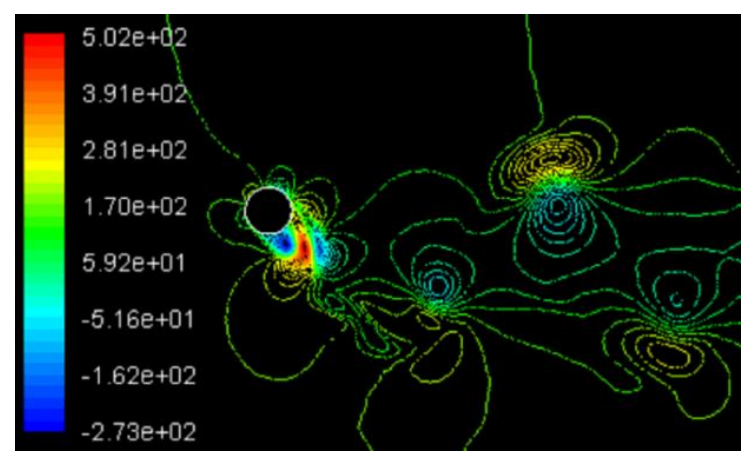

Figure 4 (a): Vortex shedding pattern behind cylinder with $\eta_{b}=1$ showing $2 S$ mode

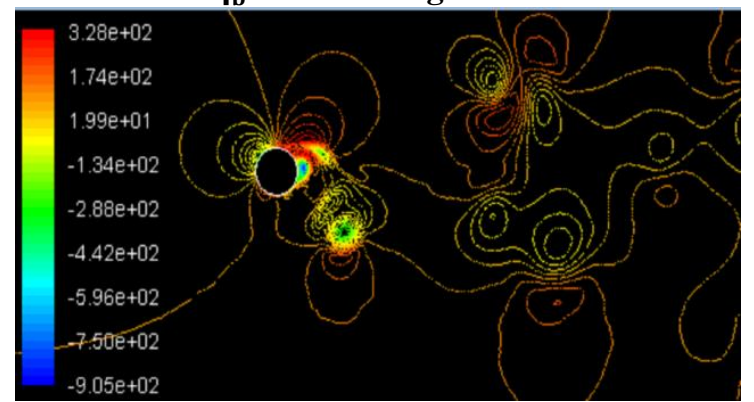

Figure 4 (b): Vortex shedding pattern behind cylinder with $\eta_{b}=2$ showing $P+S$ mode

The structural behavior of the cylinder during the two cases can be related to the vortex shedding pattern in its wake. The shedding pattern is shown in Fig 4 . For $\eta_{\mathrm{b}}=$ 1 during which a uniform variation of force coefficients and oscillation amplitude are observed the shedding pattern shows a $2 \mathrm{~S}$ mode. That is alternate vortices are shed during one oscillation. In this case the lift coefficient varies uniformly and the IL response is insignificant compared to $\mathrm{CF}$ response. For $\eta_{\mathrm{b}}=2$ the shedding pattern is no more $2 \mathrm{~S}$ mode, but a combination of a pair $(\mathrm{P})$ and single $(\mathrm{S})$ vortices ie $\mathrm{P}+\mathrm{S}$ mode of shedding. This phenomenon contributes to the increased drag forces on the cylinder. The specified structural parameters facilitate uniform response in both directions when the cylinder is subjected to $\mathrm{P}+\mathrm{S}$ mode of shedding.

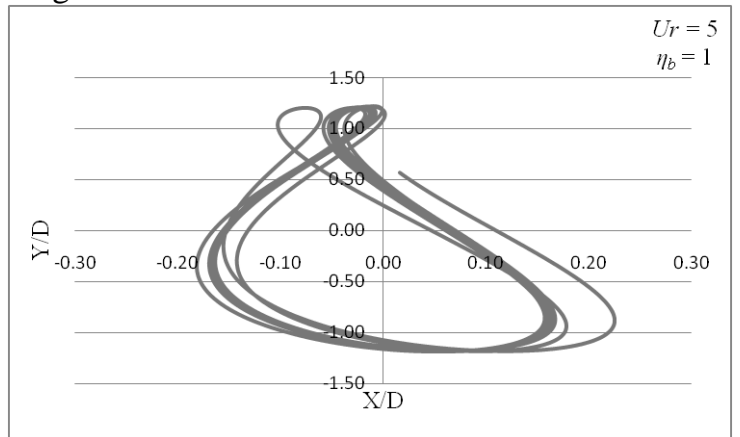

Figure 5 (a): Trajectory of the cylinder with $\eta_{b}=1$

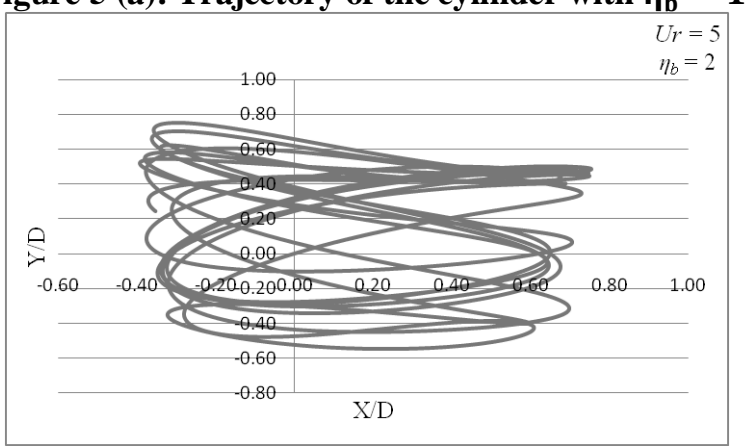

Figure 5 (b): Trajectory of the cylinder with $\eta_{b}=2$
Predicted trajectories of the cylinder for the two cases are shown in Figure 5(a) and (b). Both of them show eight figure patterns. During oscillations with $\eta_{\mathrm{b}}=1$, the cylinder follows the same spatial coordinates to complete the eight figure trajectory during each oscillation, resulting in a superimposed eight figure. But in the second case when $\eta_{\mathrm{b}}=2$, the spatial coordinates followed by the cylinder changes for each oscillation, resulting in a scattered eight figure trajectory. This observation can be attributed to the effect of IL oscillation amplitude on the overall trajectory of the cylinder. For the second case where a scattered eight figure trajectory has been observed IL and CF oscillation amplitudes are almost of same magnitude unlike the negligible magnitude of IL vibration compared to that of $\mathrm{CF}$ vibration for the first case.

\section{CONCLUSION}

Significant observation from the TDOF simulations for the two cases is that the variation of hydrodynamic force coefficients and non-dimensional response amplitudes with respect to time are observed to be uniform and regular for the first case where $\eta_{b}=1$. But for $\eta_{b}=2$ all time histories shows irregular variation. $C_{L}$ and $C_{D}$ values increases for the second case compared to the first with $\mathrm{C}_{\mathrm{L}}$ showing $18.8 \%$ increase and $\mathrm{C}_{\mathrm{D}}$ showing $34.7 \%$ increase. Frequency of oscillation of the lift force is significantly affected by the value of $\eta_{b}$. Frequency of oscillation of the cylinder in $C F$ direction remains unaffected by $\eta_{b}$, but that in the IL direction reduces and shifts to a value closer to the natural frequency of cylinder in IL direction. Cylinder is more prone to lock in vibration in both directions when $\eta_{b}=2$, since the oscillation frequency is very close to the natural frequency of the cylinder in the respective directions. A $\mathrm{P}+\mathrm{S}$ mode of shedding is also observed at $\eta_{\mathrm{b}}=2$ which indicates possibility of lock in vibration rather than the $2 \mathrm{~S}$ mode observed at $\eta_{b}=1$. From the trajectories it is evident that the amplitudes of oscillation in CF and IL directions have comparable magnitude when $\eta_{b}=2$ unlike the negligible magnitude of IL vibration compared to CF vibration when $\eta_{\mathrm{b}}=1$. The superimposed and scattered eight figure trajectories obtained in the two cases throws light on the influence of IL oscillation amplitude on the overall path followed by the cylinder during VIV.

\section{REFERENCES}

1. Khalak, A. and Williamson, C. H. K. Investigation of the relative effects of mass and damping in vortex induced vibration of a circular cylinder, Journal of Wind Engineering. Ind. Aerodyn. 69-71 (1997) 341 - 350

2. Jauvtis, N. and Williamson, C. H. K. The effect of two degrees of freedom on vortex-induced vibration at low mass and damping. Journal of Fluid Mechanics 509, (2004) 23 - 62.

3. Moe, G. and $\mathrm{Wu}, \mathrm{Z}$. J. The lift force on a cylinder vibrating in a current. Journal of Offshore Mechanics and Arctic Engineering 112 (1990) 297- 303.

4. Sarpkaya, T. Hydrodynamic damping, flow-induced oscillations, and biharmonic response. ASME Journal of Offshore Mechanics and Arctic Engineering. 117 (1995) 232-238.

5. Yin, D. Experimental and Numerical Analysis of Combined In-line and Cross-flow Vortex Induced Vibrations. Ph D Thesis, Norwegian University of Science and Technology. 2013.

6. Narendran, K., Murali, K., Sundar, V., Vortex-induced vibrations of elastically mounted circular cylinder at $\mathrm{Re}$ of the $\mathrm{O}$ $\left(10^{5}\right)$. Journal of Fluids and Structures. 54 (2015) 503 521.

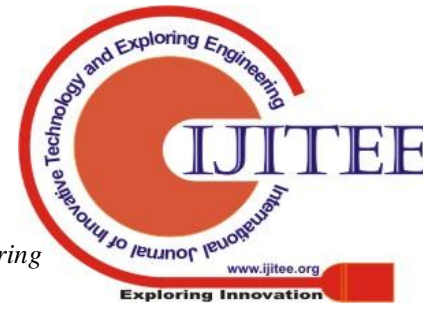


7. Naudascher, E. and Rockwell, D. Flow induced vibration - An engineering guide. Dover Publications Inc, Mineola, New York, USA, 2005.

8. Gabbai, R. D. and Benaroya, H. An overview of modeling and experiments of vortex-induced vibration of circular cylinders. Journal of Sound and Vibration, 282(3-5) (2005) 575-616.

9. Sekar, M., Srinivas, J., Kotaiah, K. R., Yang, S, H., Stability analysis of turning process with tailstock-supported workpiece, Int J Adv Manuf Technol, 43( 9-10) (2009) 862-871.

10. Sekar, M., Kantharaj, I., Savale A.S, Machining Chatter Analysis for High Speed Milling Operations 2017 IOP Conf. Ser.: Mater. Sci. Eng. 247012014.

11. Schlichting, H. Boundary layer theory. McGraw-Hill Book Company, New York, 1979.

12. Chandran, V., Sekar, M., Janardhanan, S., Menon, V., A numerical study on the influence of mass and stiffness ratios on the vortex induced motion of an elastically mounted cylinder for harnessing power. Energies. 11 (2018) 2580.

13. Chandran, V., Janardhanan, S., Sekar, M., Prediction of Hydro-dynamic Force Coefficients and Shedding Frequency during Vortex Shedding Behind a Stationary Cylinder. International Journal of Structural Mechanics and Finite Elements. 4(1) (2018).

14. Jie, W., Halvor, L., Larsen, M. L., Stergios, L., Rolf. B.

Vortex-induced vibration of a flexible cylinder: Interaction of the in-line and cross-flow responses. Journal of Fluids and Structures 63 (2016) 238-258.

\section{AUTHORS PROFILE}

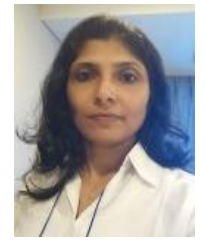

Vidya Chandran is a $\mathrm{PhD}$ scholar in the department of Mechanical Engineering, Karunya Institute of Technology and Sciences, Coimbatore, India. She has M.S (honors) from Peoples Friendship University, Russia with specialization Steam and Gas Turbines. Also she holds the position of Assistant Professor in the Department of Mechanical Engineering, SCMS School of Engineering and Technology, Ernakulum, Kerala. She served as the organizing secretary for two editions of the International Conference on Computing in Mechanical Engineering (ICCME). Research interests include Vortex Induced Vibration (VIV), renewable energy and underwater biomimetic propulsion. She has several indexed publications in these fields. She is also the co investigator of the project, Hydro Vortex Power Generator (HVPG) supported by Energy Management Centre, Government of Kerala.

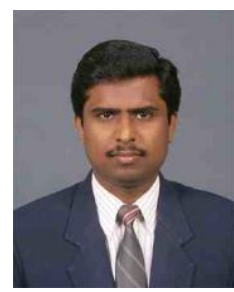

Dr. M Sekar holds the current position of Principal, AAA college of Engineering and Technology, Sivakasi, India. Formerly he held the post of Head of the Department, Mechanical Engineering at Karunya Institute of Engineering and Sciences, Coimbatore. Dr. Sekar had obtained his doctoral degree from Kyungpook National University, South Korea. He was awarded the prestigious Korean Research Fund (KRF) to carry out his research. His research interests include Computational geometry, motion control of CNC and robots, stability analysis of machining processes and industrial IOT. He is a fellow of Institution of Engineers and active member of several professional bodies. He has published more than 70 research papers in reputed International Journals and Conferences.

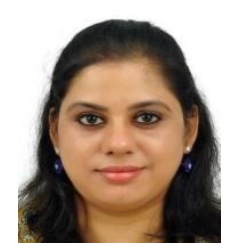

Dr. Sheeja Janardhanan is a Professor in the department of Mechanical Engineering, SCMS School of Engineering and Technology, Ernakulum, India. She has a $\mathrm{PhD}$ in Numerical Ship Hydrodynamics from the department of Ocean Engineering, Indian Institute of Technology Madras, India. Formerly she worked as Surveyor in the Research and Rule Development Division of Indian Register of Shipping, Mumbai, India. She is also the principal investigator of the project, Hydro Vortex Power Generator (HVPG) partly funded by the Energy Management Center, Govt. of Kerala, India. She is presently the organizer of the special session 'Advance Scientific Visualization For Multidisciplinary Engineering' in the international conference 'Advanced Computational Engineering and Experimenting (ACEX) which is held annually in Europe. She is also the Editor of Visual Computing-Advancing Engineering Practice, Lecture Notes in Mechanical Engineering, Springer. 